\title{
XMM-Newton observation of the Coma Galaxy cluster $^{\star, \star \star}$
}

\section{The temperature structure in the central region}

\author{
M. Arnaud ${ }^{1}$, N. Aghanim ${ }^{2}$, R. Gastaud ${ }^{3}$, D. M. Neumann ${ }^{1}$, D. Lumb ${ }^{4}$, U. Briel ${ }^{5}$, B. Altieri ${ }^{6}$,

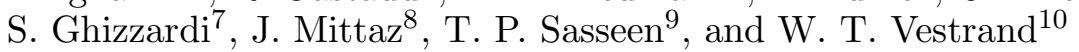 \\ 1 CEA/DSM/DAPNIA Saclay, Service d'Astrophysique, L'Orme des Merisiers, Bât. 709, \\ 91191 Gif-sur-Yvette, France \\ 2 IAS-CNRS, Université Paris Sud, Bât. 121, 91405 Orsay Cedex, France \\ 3 CEA/DSM/DAPNIA Saclay, Service d'Électronique et d'Informatique, 91191 Gif-sur-Yvette, France \\ 4 Space Science Dept., European Space Agency, ESTEC Postbus 299, 2200 AG Noordwijk, The Netherlands \\ 5 Max-Planck-Institut für extraterrestrische Physik, 85740 Garching, Germany \\ 6 XMM-Newton Science Operations Centre, ESA Space Science Department, PO Box 50727, \\ 28080 Madrid, Spain \\ 7 IFC/CNR, Via Bassini 15, 20133 Milano, Italy \\ 8 Department of Space and Climate Physics, UCL, Mullard Space Science Laboratory, Holmbury St. Mary, \\ Surrey, UK \\ 9 University of California, Santa Barbara, CA 93110, USA \\ 10 NIS-2, MS D436, Los Alamos National Laboratory Los Alamos, NM 87545, USA
}

Received 2 October 2000 / Accepted 2 November 2000

\begin{abstract}
We present a temperature map and a temperature profile of the central part $\left(r<20^{\prime}\right.$ or $1 / 4$ virial radius) of the Coma cluster. We combined 5 overlapping pointings made with XMM/EPIC/MOS and extracted spectra in boxes of $3.5^{\prime} \times 3.5^{\prime}$. The temperature distribution around the two central galaxies is remarkably homogeneous $\left(r<10^{\prime}\right)$, contrary to previous ASCA results, suggesting that the core is actually in a relaxed state. At larger distance from the cluster center we do see evidence for recent matter accretion. We confirm the cool area in the direction of NGC 4921, probably due to gas stripped from an infalling group. We find indications of a hot front in the South West, in the direction of NGC 4839, probably due to an adiabatic compression.
\end{abstract}

Key words. galaxies: intergalactic medium - Cosmology: observations - Cosmology: dark matter Cosmology: large-scale structure of the Universe - X-rays: general

\section{Introduction}

Numerical simulations of cluster evolution (e.g. Evrard 1990; Schindler \& Müller 1993) suggest that the temperature structure of the Intra-Cluster Medium (ICM) is a

Send offprint requests to: M. Arnaud,

e-mail: marnaud@discovery.saclay.cea.fr

* Based on observations obtained with XMM-Newton, an ESA science mission with instruments and contributions directly funded by ESA Member States and the USA (NASA).

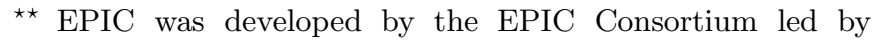
the Principal Investigator, Dr. M. J. L. Turner. The consortium comprises the following Institutes: University of Leicester, University of Birmingham, (UK); CEA/Saclay, IAS Orsay, CESR Toulouse, (France); IAAP Tuebingen, MPE Garching, (Germany); IFC Milan, ITESRE Bologna, IAUP Palermo, Italy. EPIC is funded by: PPARC, CEA, CNES, DLR and ASI. powerful indicator of the evolutionary state of clusters. In particular the accretion of a sub-cluster, a common phenomenon in standard hierarchical formation scenario, should manifest itself by characteristic features in the temperature map, like heated gas between the two units just before the collision.

Recent studies of the Coma cluster with the ASCA satellite (Donnelly et al. 1999; Watanabe et al. 1999) revealed complex temperature variations in this massive cluster. They were interpreted as indicative of recent mergers, confirming earlier evidence based on optical dynamical studies (Colless \& Dunn 1996 and references therein) and X-ray morphological analysis (Briel et al. 1992; White et al. 1993; Vikhlinin et al. 1994, 1997). ASCA covered a broad energy band, which is essential for 
Table 1. Observations

\begin{tabular}{llllcccccc}
\hline Obs. & Rev. & RA & DEC. & MOS1 & MOS2 & \multicolumn{4}{c}{ MOS1\&2 counts } \\
& & $(\mathrm{J} 2000.0)$ & $(\mathrm{J} 2000.0)$ & $\begin{array}{c}\text { Exp. } \\
\text { Exp. }\end{array}$ & \multicolumn{2}{c}{ Expl-10] keV } & \multicolumn{2}{c}{$[5-10] \mathrm{keV}$} \\
& & & $(\mathrm{ksec})$ & $(\mathrm{ksec})$ & Source & Bkgd & Source & Bkgd \\
\hline Pc & 86 & $12^{\mathrm{h}} 59^{\mathrm{m}} 47^{\mathrm{s}}$ & $27^{\circ} 57^{\prime} 00^{\prime \prime}$ & 16.4 & 16.3 & $7.7010^{5}$ & $4.210^{4}$ & $3.5210^{4}$ & $1.0010^{4}$ \\
P5 & 86 & $12^{\mathrm{h}} 59^{\mathrm{m}} 28^{\mathrm{s}}$ & $27^{\circ} 46^{\prime} 53^{\prime \prime}$ & 20.9 & 21.4 & $6.6310^{5}$ & $5.510^{4}$ & $3.2310^{4}$ & $1.3010^{4}$ \\
P6 & 93 & $12^{\mathrm{h}} 58^{\mathrm{m}} 50^{\mathrm{s}}$ & $27^{\circ} 58^{\prime} 52^{\prime \prime}$ & 7.4 & 7.3 & $1.8110^{5}$ & $1.910^{4}$ & $8.5310^{3}$ & $4.5210^{3}$ \\
P9 & 93 & $13^{\mathrm{h}} 00^{\mathrm{m}} 33^{\mathrm{s}}$ & $27^{\circ} 56^{\prime} 59^{\prime \prime}$ & 20.8 & 21.0 & $7.0310^{5}$ & $5.410^{4}$ & $3.0810^{4}$ & $1.2910^{4}$ \\
P10 & 98 & $12^{\mathrm{h}} 59^{\mathrm{m}} 38^{\mathrm{s}}$ & $28^{\circ} 07^{\prime} 40^{\prime \prime}$ & 20.6 & 20.9 & $5.2610^{5}$ & $5.410^{4}$ & $2.4310^{4}$ & $1.2710^{4}$ \\
\hline
\end{tabular}

precise temperature estimate, but the observations suffered from a relatively large energy dependent PSF. Therefore temperature structure determination with ASCA might have been subject to systematic errors. Furthermore the spatial resolution was insufficient to resolve precisely the temperature radial profile in the very core of clusters.

The EPIC instrument (Turner et al. 2001) on board XMM (Jansen et al. 2001) combines a high sensitivity with good spatial and spectral resolution, on a wide energy range. In this paper, we use this unique capability to study the temperature structure in the central $\left(\theta<20^{\prime}=0.78 \mathrm{Mpc}\right)$ region of Coma. We present further $\mathrm{XMM}$ results in two other papers of this issue: the large scale morphology of Coma (Briel et al. 2001) and the dynamics of the infalling NGC 4839 group (Neumann et al. 2001). In the following, we assume $H_{0}=50 \mathrm{~km} \mathrm{~s}^{-1} / \mathrm{Mpc}$ and $q_{0}=0.5$.

\section{Data analysis}

\subsection{The data}

The central part of Coma was observed with 5 overlapping pointings in Full Frame mode with the EPIC/MOS camera (medium filter) and in extended Full Frame mode with the pn camera. As CTE correction in this pn mode is still being studied, we considered only MOS data in the present spectroscopic analysis.

We generated calibrated event files with SASv4.1, except for the gain correction. Correct PI channels are obtained by interpolating gain values obtained from the nearest observations of the on board calibration source. Data were also checked to remove any remaining bright pixels. We excluded periods of high background induced by solar flare protons. We discarded all frames corresponding to a count rate greater than $15 \mathrm{ct} / 100 \mathrm{~s}$ in the [10-12] keV band, where the emission is dominated by the particle induced background. Finally, only events in the nominal FOV are considered.

The central position of each pointed observation is listed in Table 1, together with the revolution number and remaining observing time after cleaning.

\subsection{Spatially resolved spectroscopy}

Spectra in various regions were considered to study temperature variations. Each pointing and each MOS camera are first treated separately.

\subsubsection{Correction for vignetting effects}

The effective area at a given energy depends on position. When extracting the spectrum of a region, we weight each photon falling at position $\left(x_{j}, y_{j}\right)$ of the detector and of energy $E_{j}$ by the ratio of the effective area at that position, to the central effective area (for this energy). This weighting is taken into account in the error estimate. The "corrected" spectrum obtained is an estimate of the spectrum one would get if the detector was flat. A detailed description of the method and of the vignetting calibration data used are given in Arnaud et al. (2001). Note that the vignetting due to the RGA is included, but is assumed to be energy independent (the variations are less than $1 \%$ below $6 \mathrm{keV})$.

\subsubsection{Background estimate}

We generated EPIC MOS background event files (one for each MOS camera) by combining several high galactic latitude pointings. The data are cleaned for bright pixels, background flares and regions corresponding to bright point sources are excluded. The integrated exposure time is $94.3 \mathrm{ksec}$ for MOS1 and $78.9 \mathrm{ksec}$ for MOS2. These event files can be used for a proper estimate of the cosmic ray (CR) induced background but not the X-ray background, which depends on pointing position and filter. However, bright cluster emission, like the one observed in the Coma center, usually dominates the background except at high energy (see also Arnaud et al. 2001). We thus are mostly sensitive to $\mathrm{CR}$ induced background. Note also that the offset pointings considered here always include the cluster peak emission, so scattered light is not a problem. The total estimated number of source and background photons, in the $[0.3-10] \mathrm{keV}$ and $[5-10] \mathrm{keV}$ energy ranges, are listed in Table 1 for each pointing. Note the degradation of the $\mathrm{S} / \mathrm{N}$ ratio at high energies as a consequence of the very hard $\mathrm{CR}$ induced background. 


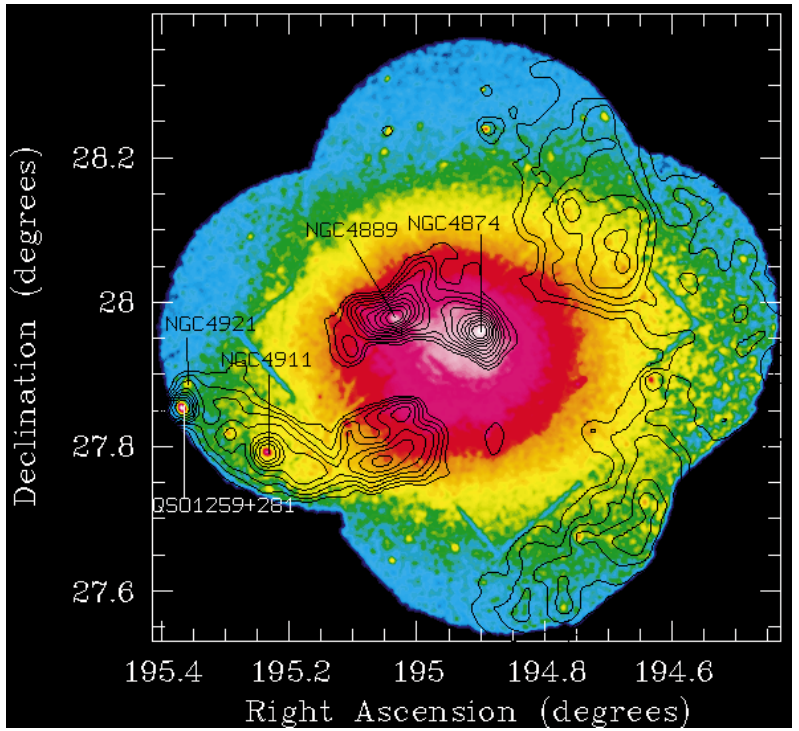

Fig. 1. The EPIC/MOS mosaic image of the central region of Coma (5 overlapping pointings) in the [0.3-2] energy band. The iso contours are the residuals (in $\sigma$ ) after subtracting the best fit $2-\mathrm{D} \beta$ model. The step size is $4 \sigma$ and the lowest isocontour corresponds to $3 \sigma$ significance. The position of the bright galaxies are marked

It is known that the CR background changes slightly in the FOV. It is thus better to consider the same extraction regions in detector coordinates for the source and the background. Furthermore, if one wants to combine spectra of a given physical Coma region obtained from different pointings, it is mandatory to define extraction regions in sky coordinates. To alleviate this practical problem, we simply generated a specific background event file for each Coma pointing and camera by modifying the sky coordinates of the background event file using the aspect solution of the considered Coma observation.

For consistency, the background spectra were obtained using the same correction method as used for the source. The background component induced by $\mathrm{CR}$ is not vignetted, but as we extract the background and source spectra from the same region in detector coordinates the correction factor is the same and does not introduces bias.

Further details on the characteristic of the EPIC/MOS background and subtraction method can be found in Arnaud et al. (2001).

\subsubsection{Spectra extraction and spectral fitting}

The source and background spectra of a given region (defined in sky coordinates) are first extracted separately for each MOS camera and each pointing, using the method described above. As the spectra are corrected for vignetting, the spectra of the same physical region observed with different off-axis angle (from different pointings) and different camera can be simply added to maximize the signal to noise ratio. The errors are propagated using quadratic summation.

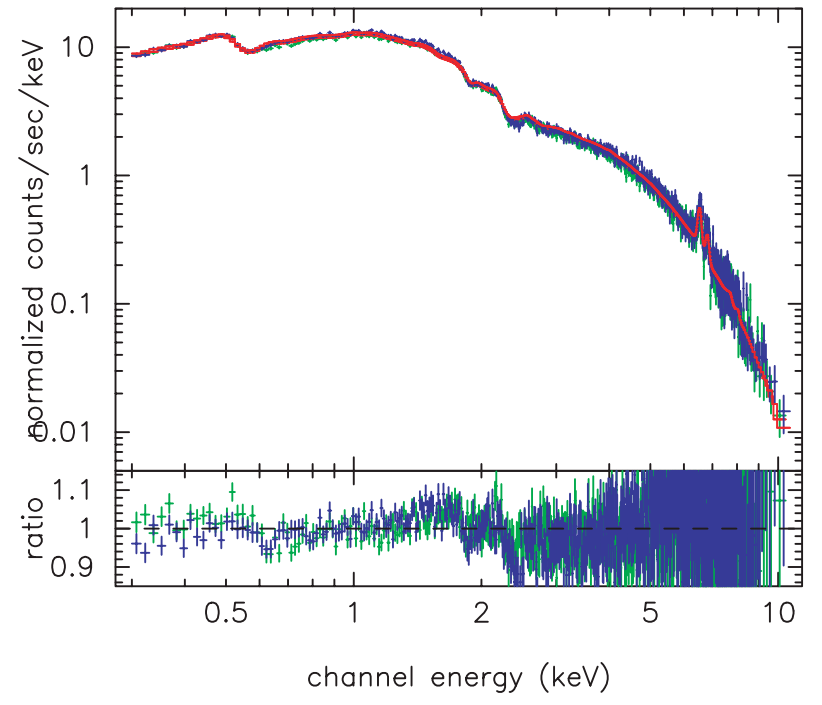

Fig. 2. EPIC/MOS1 (green) and EPIC/MOS2 (blue) spectra extracted from within $10^{\prime}$ in radius of the galaxy NGC 4874 . Red line: best fit redshifted isothermal model: $k T=8.25 \mathrm{keV}$ and an abundance of 0.25 . Bottom panel: residuals between model and data

Before model fitting, the source spectra are binned so that the $\mathrm{S} / \mathrm{N}$ ratio is greater than $3 \sigma$ in each bin after background subtraction. The spectra are fitted with XSPEC using isothermal mekal models (with fixed redshift $z=0.0231$ ). Although the thermodynamic state of the plasma could be more complex (e.g. see the isobaric multiphase model of Nagai et al. 2000), this is adequate to study temperature spatial variations, the derived best fit temperature being an estimate of the mean temperature in each projected region considered. Since the spectra are "corrected" for vignetting effects we can use the on axis MOS response file, which is considered to be the same for MOS1 and MOS2 (version v3.15). Only data above $0.3 \mathrm{keV}$ are considered due to remaining uncertainties in the MOS detector response below this energy. Unless otherwise stated errors are with a $90 \%$ confidence level.

\subsection{Imaging analysis}

The MOS mosaic image in the $[0.3-2] \mathrm{keV}$ energy band is presented in Fig. 1. The count images for each camera and each pointing are extracted using the weighting procedure described above to correct for vignetting. They are then projected on a common sky reference axis, summed and divided by the mosaic exposure map (which takes into account the exposure time and FOV coverage for each pointing). The images are not background subtracted. In that energy range and in this central area of the cluster, the particle background is negligible for MOS. 


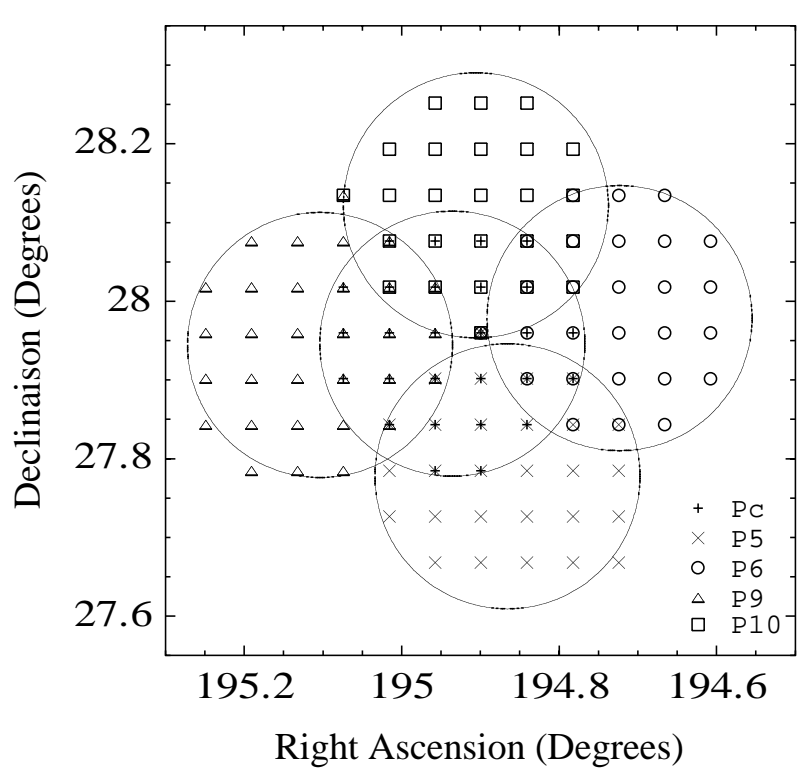

Fig. 3. Position of the $3.5 \times 3.5^{\prime}$ boxes, in which we derive EPIC/MOS spectra. A different symbol is used for each of the 5 pointed observations. When the same region is observed in two or more pointings, the corresponding spectra are summed (see text). The spectrum of each region is fitted with an isothermal model to build the temperature map displayed in Fig. 5. Circles: central $\theta<10^{\prime}$ FOV of each pointed observation

\section{Results}

\subsection{Core morphology}

To identify significant substructures in the core, we fitted the MOS image with a $2-D$ ellipsoidal $\beta$ model plus background and built up the map of the residuals of the data over the best fit model. The method is discussed in detail in Neumann \& Böhringer (1997) and Neumann (1999). The iso-contours of significance of the excess (number of $\sigma$ over background plus cluster model) are overlaid on the MOS image in Fig. 1.

We unambiguously confirm the excess emission around the two central galaxies (NGC 4874 and NGC 4889) and the tail of gas in the direction of NGC 4911, revealed by the wavelet analysis of Vikhlinin et al. (1997). However, contrary to Vikhlinin et al. result, this filamentary structure does not seem to be directly connected to the Coma center, but originates $0.5 \mathrm{Mpc}$ South of it. Note also that part of the excess is due to resolved galaxy emission (NGC 4911, NGC 4921, QSO 1259+281, and 5 other point sources).

Diffuse excess emission is clearly detected in the SouthWest in particular in the direction of the NGC 4839 group (see Briel et al. 2001, for full discussion).

\subsection{Overall spectrum}

To compare with results obtained with other satellites we extracted the overall MOS1 and MOS2 spectra from a circular region of 10 arcmin in radius centered on NGC 4874. Only data from the central pointing are used. The data

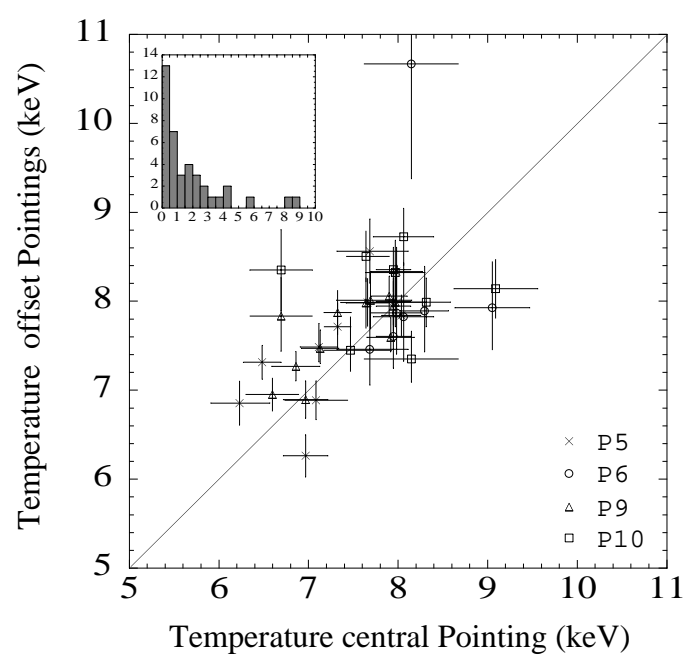

Fig. 4. Temperature determined from the offset pointings (P5, P6, P9 and/or P10) versus the temperature obtained, for the same region, using the central pointing $(\mathrm{Pc})$ data. Errors are at $1 \sigma$-level. The insert shows the histogram of the differences in term of $\chi^{2}$ (see text for details)

are fitted in the $[0.3-10] \mathrm{keV}$ range, with independent normalizations for MOS1 and MOS2, and common temperatures and abundances. The spectra are plotted in Fig. 2, the bottom panel gives the residuals. Fixing the $N_{\mathrm{H}}$ value to the $21 \mathrm{~cm}$ value $\left(N_{\mathrm{H}}=8.9510^{19} \mathrm{~cm}^{-2}\right.$, Dickey \& Lockman 1990), we obtain a best fit temperature of $k T=8.25 \pm 0.10 \mathrm{keV}$ and an abundance of $0.25 \pm 0.02$. The reduced $\chi^{2}$ is 1.38 ( $\chi^{2}=1457$ for 1058 d.o.f). The fit is satisfactory. The residuals are concentrated around the instrument edges, with residual ratios between data and model of about $\pm 5 \%$, consistent with our present knowledge of the instrument response (Fig. 2). If we let the $N_{\mathrm{H}}$ value free we get $k T=8.20 \mathrm{keV}$, an abundance of 0.25 and $N_{\mathrm{H}}=9.4 \pm 0.910^{19} \mathrm{~cm}^{-2}$, in agreement with the $21 \mathrm{~cm}$ value and $\chi^{2}$ is unchanged. In the following we thus fix the $N_{\mathrm{H}}$ value to the $21 \mathrm{~cm}$ value.

When fitted separately the MOS1 and MOS2 temperatures are consistent and the relative normalization is 1.05 . In the following analysis we will thus sum MOS1 and MOS2 spectra.

The best fit overall temperature in this central $(R<$ $\left.10^{\prime}\right)$ region is in remarkable agreement with the overall GINGA value of $k T=8.21 \pm 0.09 \mathrm{keV}$ (Hughes et al. 1993) and is marginally consistent with the ASCA value of $9 . \pm 0.6 \mathrm{keV}$ (Donnelly et al. 1999) obtained for the central $\left(R<9^{\prime}\right)$ region. Note that the hard excess seen by SAX (Fusco-Femiano et al. 1999) could not be seen by $\mathrm{XMM}$, since it appears above $20 \mathrm{keV}$.

\subsection{Temperature map}

To study the cluster temperature structure, we next extracted spectra in $3.5^{\prime} \times 3.5^{\prime}$ contiguous regions in sky coordinates. The box size was chosen so that the two central galaxies fall approximately in the center of a box, 


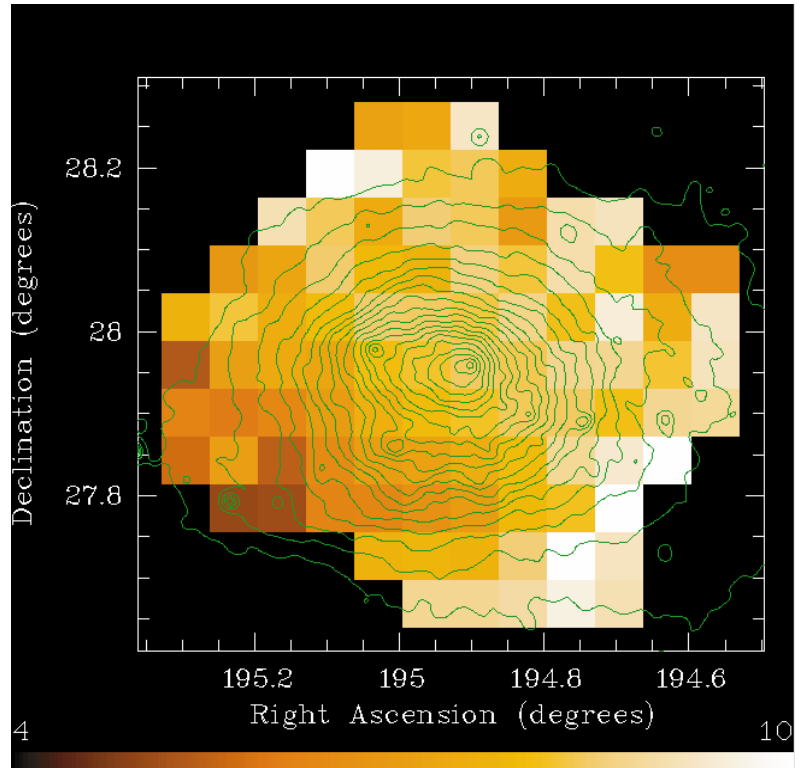

Fig. 5. Color coded temperature map. Note the hot front in the south-west (white) and the cold region in the South-East (brown/dark red). The isocontours of the PN image in the [0.3-2.] keV band (Briel et al. 2001) are superimposed. The lowest contour corresponds to $6.310^{-3} \mathrm{ct} / \mathrm{s} / \mathrm{arcmin}^{2}$ and the step size is $410^{-3} \mathrm{ct} / \mathrm{s} / \operatorname{arcmin}^{2}$

and that a sufficient $\mathrm{S} / \mathrm{N}$ ratio is reached for each box. Circular regions ( $20^{\prime \prime}$ in radius) around bright sources (in particular NGC 4889 and NGC 4911) are excised from the boxes. The overall region considered for this spatially resolved spectroscopic analysis is about $20^{\prime}$ in radius. We only considered boxes at off-axis angles smaller than $10^{\prime}$ in each pointing (the vignetting factor being more uncertain beyond this radius). The central $\theta<10^{\prime}$ region of each pointing, delineated as circle, and the central position of the various boxes (95 in total) are plotted in Fig. 3.

The validity of our vignetting correction can be assessed by comparing the fitted temperature of the same region in various pointings. The vignetting effect (decrease of effective area with off-axis angle) increases with energy. An understimate (overestimate) of this energy dependence would yield to underestimate (overestimate) of the temperature. Since the various pointings of the same region correspond to different off-axis angles, an improper vignetting correction would translate in systematic differences between temperature estimates for the same region. Figure 4 shows the temperatures determined from the offset pointings $T_{\text {off }}$ versus the temperature obtained, for the same region, using the central pointing $T_{\mathrm{c}}$ (errors are at $1 \sigma$-level). The insert shows the histogram of the differences in term of $\chi^{2}=\left(T_{\text {off }}-T_{\mathrm{c}}\right)^{2} /\left(\sigma\left(T_{\text {off }}\right)^{2}+\sigma\left(T_{\mathrm{c}}\right)^{2}\right)$ computed for each pair of measurements. Three outliers ( $\chi^{2}>4$ or more than $2 \sigma$ discrepancy in estimates) are clearly apparent. They correspond to the points at $(6.7$, $8.4),(6.5,7.3)$ and $(7.6,8.5)$. We do not see any particular problem in the corresponding spectra (the statistic and fit are good) and failed to find any obvious reason

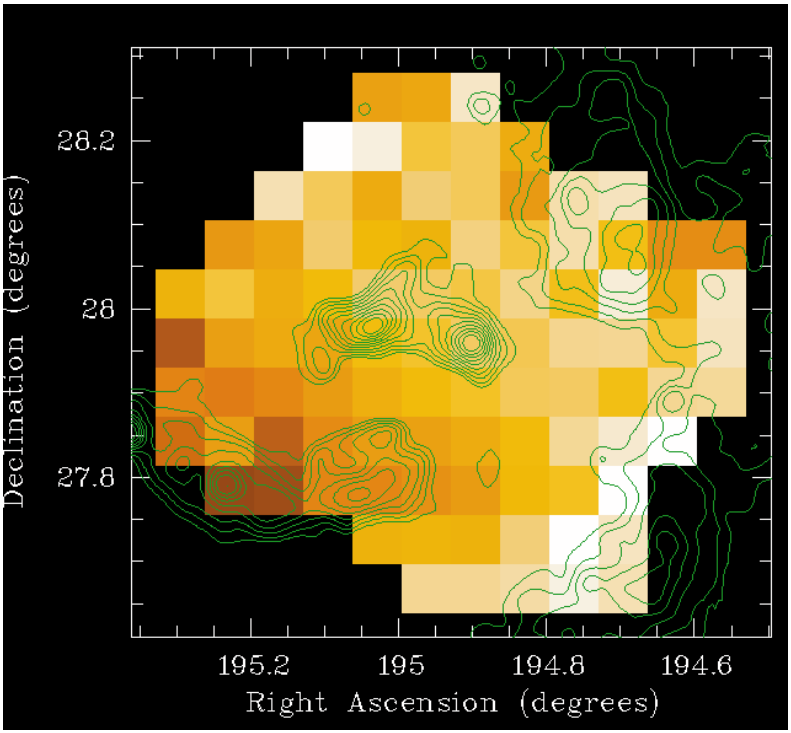

Fig. 6. Excess emission over a $\beta$ model overlayed on the temperature map. Isocontours are as in Fig. 1

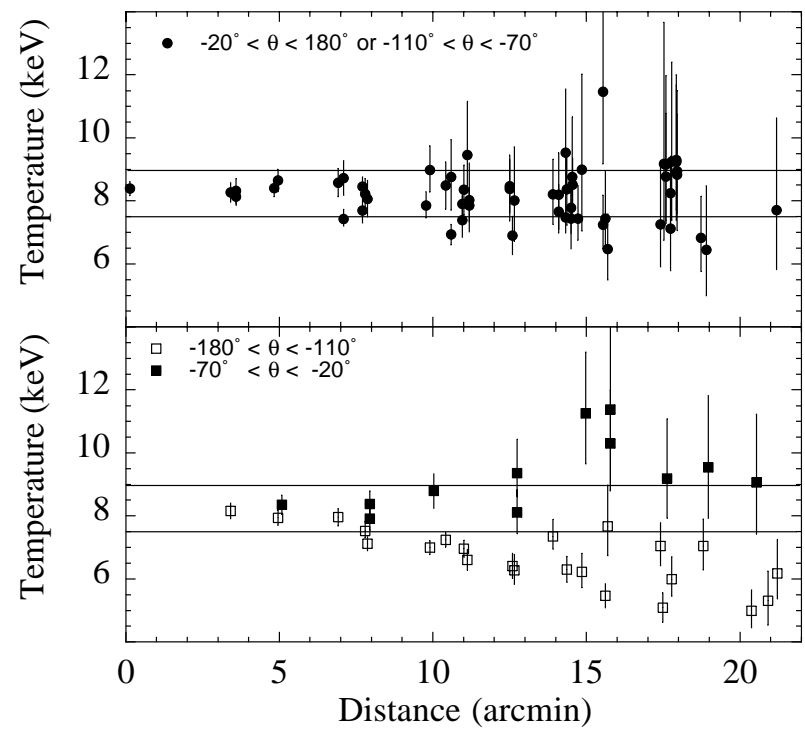

Fig. 7. Temperature of each region of the temperature map with $90 \%$ confidence error bars. The temperature is plotted versus the distance to the brightest central galaxy NGC 4874 and the data are splitted in three subsamples. Bottom panel: the S-E sector encompassing the filamentary structure towards NGC 4911 (open squares) and the S-W sector along the direction towards NGC 4839 group (filled squares). Top panel: the rest of the regions (filled circles). The angle (counterclockwise from west) defining the sectors are indicated in the figure

for the discrepancy. However, these outliers correspond to isolated regions (the agreement between estimates is good in adjacent regions) and the overall $\chi^{2}=44$ for 36 d.o.f is satisfactory when these outliers are excluded. This suggests that the vignetting correction is basically correct. We thus sum all spectra obtained for a given physical region and build up the temperature map presented in Fig. 5.

There is no strong evidence of temperature variations, except for a cold area in the South-East (contiguous 


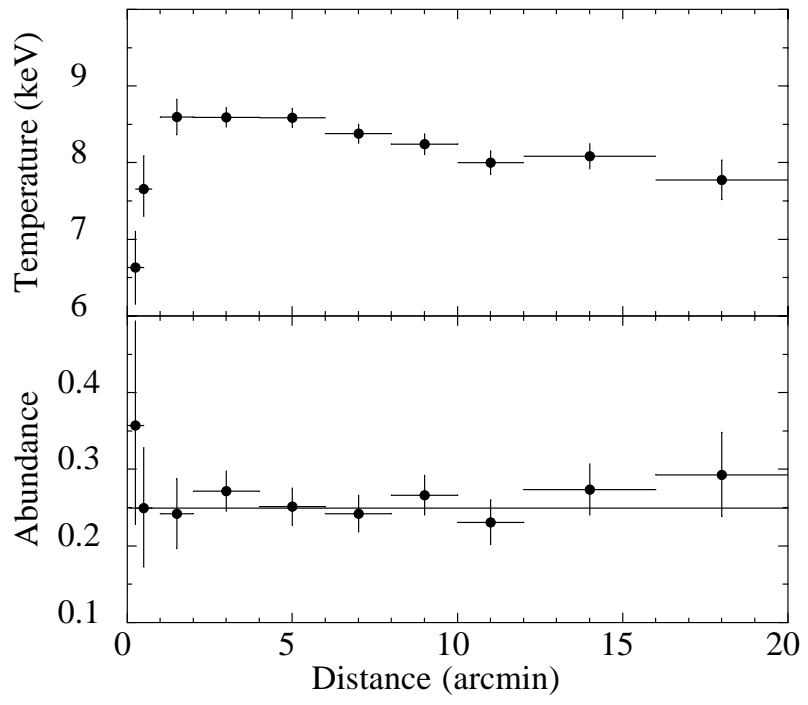

Fig. 8. Radial temperature (top panel) and abundance (bottom panel) profiles. The rings are centered on NGC 4874

regions colder than average) and a hot area in the SouthWest. It is instructive to compare these temperature features with the X-ray image substructures (Fig. 6). The $\mathrm{S} / \mathrm{E}$ cold region in the temperature map generally coincides with the filamentary substructure originating near NGC 4911 and NGC 4921. It includes the cold regions put into evidence by Donnelly et al. (1999) in that area (region 1 and part of region 20 in their Fig. 2), that we thus confirm. The hot regions in the $\mathrm{S} / \mathrm{W}$ appear as a hot front perpendicular to the direction connecting the cluster center to the NGC 4839 group, just ahead of the excess emission in that direction. This excess emission extends somewhat further to the North, where no specific temperature feature is apparent. However, the temperature map is specially noisy in that direction. The statistical significance of the temperature variations can be seen in Fig. 7 where we plotted the temperature of the various boxes versus their distance to NGC 4874. We split the data in three subsamples: i) one S-E sector encompassing the filamentary structure towards NGC 4911 ii) one S-W sector along the direction towards NGC 4839 group iii) the rest of the sample. The hot front $(k T \sim 11 \mathrm{keV})$ in the $\mathrm{S}-\mathrm{W}$ located at about $15^{\prime}$ from the center clearly stands out, as well as the colder region $(k T \sim 6 \mathrm{keV})$ beyond $10^{\prime}$ in the $\mathrm{S}-\mathrm{E}$ sector. Otherwise the temperature does not deviate significantly from the $8.25 \pm 0.75 \mathrm{keV}$ temperature range (less than $\pm 9 \%$ variation). In particular, we see no evidence of the hot spot seen by Donnelly et al. (1999) $3^{\prime}$ north of the NGC 4874 galaxy. We further extracted the spectrum (using the central pointing) corresponding to this hot ASCA region: from Fig. 1 of Donnelly et al. (1999) we considered a rectangular region of size $7.4^{\prime} \times 3.2^{\prime}$ centered on $\alpha=12^{\mathrm{h}} 59^{\mathrm{m}} 40^{\mathrm{s}}, \delta=28^{\circ} 00^{\prime} 30^{\prime \prime}$. We get $k T=8.4 \pm 0.4 \mathrm{keV}$ consistent with the mean value and inconsistent with the ASCA value of Box $11\left(k T=12.7_{-2.0}^{+3.6} \mathrm{keV}\right)$.

\subsection{Temperature profile}

We further extracted spectra in concentric rings centered on NGC 4874 (all the data are summed). The region around NGC 4889 (40" in radius) and bright point sources were excluded. The temperature and abundance profiles are shown in Fig. 8.

\section{Discussion and conclusion}

The dynamical state of the core of Coma has been much debated, in particular the nature of the merging unit(s) and their link with the dominant cluster galaxies. If there is a consensus that a merging group is associated with NGC 4889, the situation of NGC 4874 is less clear (see in particular Colless \& Dunn 1996; Donnelly et al. 1999). As already proposed by Colless \& Dunn (1996), our data suggest that NGC 4874 is simply the central galaxy of the main Coma cluster, rather than being associated with a second subgroup in an early merging stage with the main cluster (as proposed by Donnelly et al. 1999). Several facts support this picture. First the remarkably homogeneous temperature distribution within the central $\theta<10^{\prime}$ region suggests the gas in that region is basically in a relaxed state. Second, there is no obvious evidence of a third peak in between NGC 4889 and NGC 4874, which would be associated with the "true" cluster center. Actually, apart from the excess around NGC 4889, the X-ray morphology can be classified as an offset-center cluster morphology (variation of isophote centroid with scale). The excess (size $\sim 3^{\prime}$ in radius) around NGC 4874 , when subtracting a $\beta$ model (representative of the large scale morphology) is a natural consequence for this type of morphology. Moreover, part of the excess is certainly due to the contribution of the halo of the galaxy itself. The very significant drop in temperature within $1^{\prime}=40 \mathrm{kpc}$ of NGC 4874 (Fig. 8) is natural in that context, as well as the increase in abundance, which could be due to an enriched ISM.

It might be surprising that substructures survive in the gas density distribution (excess around NGC 4889 and centroid shift for the main cluster) while the temperature distribution appears homogeneous. We must first emphasize that the spatial resolution and accuracy of the temperature and imaging data is not comparable. However, our results may also indicate that the gas simply follows the dark matter distribution. Dark matter substructures can survive for a long time after mergers, as indicated by high resolution simulations (Moore et al. 1998). High resolution hydrodynamic simulation, and more sophisticated morphology analysis, are essential to better understand this issue.

At larger scale we do see evidence of recent merger activity. The cold filamentary structure in the SouthEast can be naturally explained by a merging group (see Vikhlinin et al. 1997; Donnelly et al. 1999). The position and extent of the cold substructure and the core properties outlined above suggest that the merging group is associated with NGC 4911 and NGC 4921, rather than being 
due to gas stripped from a group centered on NGC 4874 as proposed by Donnelly et al. (1999). Note that an excess in the galaxy distribution is also observed around NGC 4921/NGC 4911 (Mellier et al. 1988).

Our analysis revealed for the first time a hot front in the South-West, just ahead of the excess emission that we see at the edge of the MOS mosaic and which extends further away towards NGC 4839 (see Briel et al. 2001). It is situated roughly at the boundary of the group associated with this galaxy, as defined from the optical (Colless \& Dunn 1996, Fig. 9) and is perpendicular to the direction connecting the center of Coma and NGC 4839. This temperature structure is likely to be due to adiabatic compression, caused by the infall of matter associated with the NGC 4839 group. The feature we find is indeed very similar to the one displayed in Fig. 6c of Schindler \& Müller (1993), where at this time of the merger event no accretion shock has yet formed. To definitively characterise the feature we need to know the temperature structure at larger radii, to quantify the transition conditions. It has already been noted that the NGC 4839 group is located along the large scale filament connecting Coma and A1367 (e.g. West et al. 1995). It is commonly thought that clusters form preferentially through anisotropic accretion of sub-clusters along large scale filaments. Our finding supports this scenario. The merger activity in that direction, particularly interesting for our understanding of cluster formation, is further discussed in Briel et al. (2001) and Neumann et al. (2001).

Except for the very center as discussed above, the abundance is constant and the temperature radial profile is very weakly decreasing with radius. The slight drop beyond $10^{\prime}$ is likely to be due to the cold $\mathrm{S} / \mathrm{W}$ structure. The implications of this profile for the distribution of dark matter in the core will be studied in a forthcoming paper.

Acknowledgements. We would like to thank J. Ballet for support concerning the SAS software and J.-L. Sauvageot for providing the gain correction. We thank S. Schindler and the anonymous referee for useful comments, which improved the paper.

\section{References}

Arnaud, M., Neumann, D. M., Aghanim, N., Gastaud, R., \& Majerowicz, S. 2001, A\&A, 365, L67

Briel, U. G., Henry, J. P., \& Böhringer, H. 1992, A\&A, 259, L31

Briel, U. G., Henry, J. P., Lumb D. H., et al. 2001, A\&A, 365, L60

Colless, M., \& Dunn, A. 1996, ApJ, 458, 435

Dickey, J. M., \& Lockman, F. J. 1990, ARA\&A, 28, 215

Donnelly, R. H., Markevitch, M., Forman, W., et al. 1999, ApJ, 513, 690

Evrard, A. E. 1990, ApJ, 363, 349

Fusco-Femiano, R., dal Fiume, D., Feretti, L., et al. 1999, ApJ, 513, L21

Hughes, J. P., Butcher, J. A., Stewart, G. C., \& Tanaka, Y. 1993, ApJ, 404, 611

Jansen, F., Lumb, D., Altieri, B., et al. 2001, A\&A, 365, L1

Mellier, Y., Mathez, G., Mazure, A., Chauvineau, B., \& Proust, D. 1988, A\&A, 199, 67

Moore, B., Governato, F., Quinn, T., Stadel, J., \& Lake, G. 1998, ApJL, 499, 5

Nagai, D., Sulkanen, M. E., \& Evrard, A. E. 2000, MNRAS, 316,120

Neumann, D. M., \& Böhringer, H. 1997, MNRAS, 289, 123

Neumann, D. M. 1999, ApJ, 512, 630

Neumann, D. M., Arnaud, M., Gastaud, R., et al. 2001, A\&A, 365, L74

Schindler, S., \& Müller, E. 1993, A\&A, 272, 137

Turner, M. J. L., Abbey, A., Arnaud, M., et al. 2001, A\&A, 365, L27

Vikhlinin, A., Forman, W., \& Jones, C. 1994, ApJ, 435, 162

Vikhlinin A., Forman W., \& Jones, C. 1997, ApJ, 474, L7

Watanabe, M., Yamashita, K., Furuzawa, A., et al. 1999, ApJ, 527,80

West, M. J., Jones, C., \& Forman, W. 1995, ApJ, 451, L5

White, S. D. M., Briel, U. G., \& Henry, J. P. 1993, MNRAS, 261, L8 